Przemysław Kuras*, Łukasz Ortyl*

\title{
Selected Geometric Aspects of Planning and Analysis of Measurement Results of Tall Building Structures Using Interferometric Radar**
}

\section{Introduction}

Ground-based interferometric radar IBIS, manufactured by the Italian company IDS, is a device used for measuring the displacements of points located in its field of view. The range of the observed area depends upon the characteristics of the antennas used. The principle of operation is based on a comparison of the phases of waves reaching the receiving antenna of the radar, reflected from the points in motion [3]. This means that displacements can only be observed in the radial direction, that is, a displacement of a point moving in a direction perpendicular to the axis of the transmitted beam will not be observed. Radar uses microwaves with a frequency of 17.20 GHz, modulated in a range of $\pm 0.15 \mathrm{GHz}$.

IBIS can be used in two systems: IBIS-L and IBIS-S. The L version (from landslides) is used to observe changes in positions of the points distributed on a certain surface. This system was applied to observe the displacements of water dams [1] and landslides [18]. The $S$ version (from structures) is used to observe the displacements of engineering structures, in particular those having elongated shapes [4]. In this version, the radar can operate in two modes: static and dynamic, so it is used to study bridge structures subjected to loads [8-11]. Thanks to its properties, it is also able to measure displacements of tall structures such as steel masts [12], industrial chimneys [16], towers of wind turbines [14] or towers of historic buildings [15]. The measurement results of the displacements, obtained using the interferometric radar, can be used for diagnostics of building structures, for example, subjected to tremors or rheological phenomena $[2,13]$.

The use of an interferometric radar for measuring displacements of engineering structures is relatively new. The measurement itself is performed in a remote

* AGH University of Science and Technology, Faculty of Mining Surveying and Environmental Engineering

** The study was the carried out with financial support from grant no. 15.11.150.242/13, AGH University of Science and Technology 
manner and it does not require a significant involvement of the observer, however, planning of the observations requires numerous factors to be taken into account. The article focuses on the elements which are essential to optimize the measurements of tall structures. The choice of the instrument position, relative to the structure, is of a particular importance, as it affects: effective resolution, precision of displacement measurements, the range of observations, and the limitation of operating parameters in the dynamic mode. The use of appropriate microwave antennas has been also discussed. A problem associated with the geometric analysis of the observations has been analyzed as well.

\section{The Importance of SNR}

Each radar device operates making use of a phenomenon of radio waves being reflected from targets located in the observed area. In the IBIS-S system, information about the elements located in the radar field of view are contained in the radar profile. It is presented as a graph in which the horizontal axis represents the distance $r$ from the radar to the structure, and the vertical axis - intensity of the echo signal, expressed as SNR [3]. This value is the ratio of the reflected signal intensity to noise, and is expressed in decibels.

a)

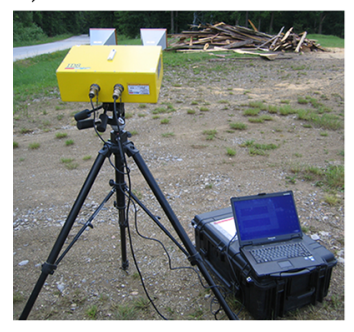

b)

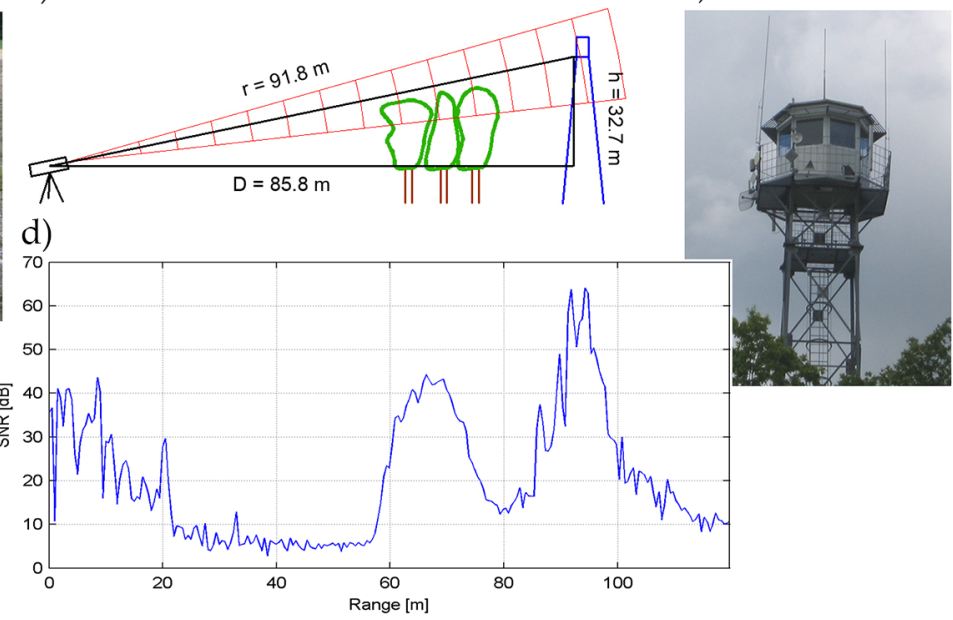

Fig. 1. Measurement of a tall structure: a) IBIS-S radar directed towards the structure;

b) geometry of the measurement; c) top of the observed tower; d) radar profile

Figure 1 depicts a situation in which the range of the observed space contains obstacles, in addition to the appropriate structure itself. Performance of interferometric radar measurements requires such instrument location that the SNR value was as large as possible. It depends upon several factors, including the material 
from which the structure is made, the intensity of the transmitted wave and the distance to the structure. Increase of the SNR value can be achieved by installing a radar reflector on the structure, which in the case of towers, may be difficult or even impossible.

According to the manufacturer [3, 15, 17], displacement measurement error, when using the interferometric radar, which should be related to the observed (or radial) direction of the displacements, can reach the value of $m_{d r}<0.1 \mathrm{~mm}$. However, this value depends on the SNR, according to Table 1 .

Table 1. The relationship between SNR and the displacement measurement error

\begin{tabular}{|c||c|c|c|c|c|c|c||}
\hline \hline SNR [dB] & 0.0 & 6.0 & 9.5 & 12.8 & 20.0 & 30.0 & 40.0 \\
\hline$m_{d r}[\mathrm{~mm}]$ & 3.39 & 1.47 & 0.95 & 0.64 & 0.28 & 0.09 & 0.03 \\
\hline \hline
\end{tabular}

Source: [18]

During the measurements of real structures, the SNR value often exceeds $30 \mathrm{~dB}$ (as in Figure 1), so in theory the displacement measurement error is very small. However, while estimating its value, other factors should be taken into account, such as the variability of weather conditions or instability of the measuring device. Based on the information from the manufacturer and the conducted field tests [5, 7], the value of $m_{d r}=0.1 \mathrm{~mm}$ was adopted for further analyses.

\section{Geometric Measurement Parameters}

Field works associated with the IBIS radar operation include several stages:

1. selection of operating mode and setting measurement parameters,

2. verification of the radar profile,

3. defining the measurement geometry,

4. performing the measurement,

5. pre-processing of the results.

The particular working stages have been described in detail in [5]. However, regarding the further part of the work, attention should be paid to several parameters, which the observer must be aware of before the measurement has been performed. These include:

- range resolution,

- projection factor,

- characteristics of the antennas.

\subsection{Range Resolution}

IBIS radar, thanks to using a special type of continuous wave frequency modulation - SFCW (stepped frequency continuous wave), has the possibility to observe 
numerous points at the same time [3]. This property is specified by the range resolution $\Delta R$ (Fig. 2). Its value depends on the frequency bandwidth used by the radar. In the case of IBIS radar, the maximum bandwidth is $300 \mathrm{MHz}$, which translates into $0.5 \mathrm{~m}$ of the range resolution. This value of $\Delta R$, as the maximum one, i.e. which provides the most detailed image of the structure, will be used for further analyses.

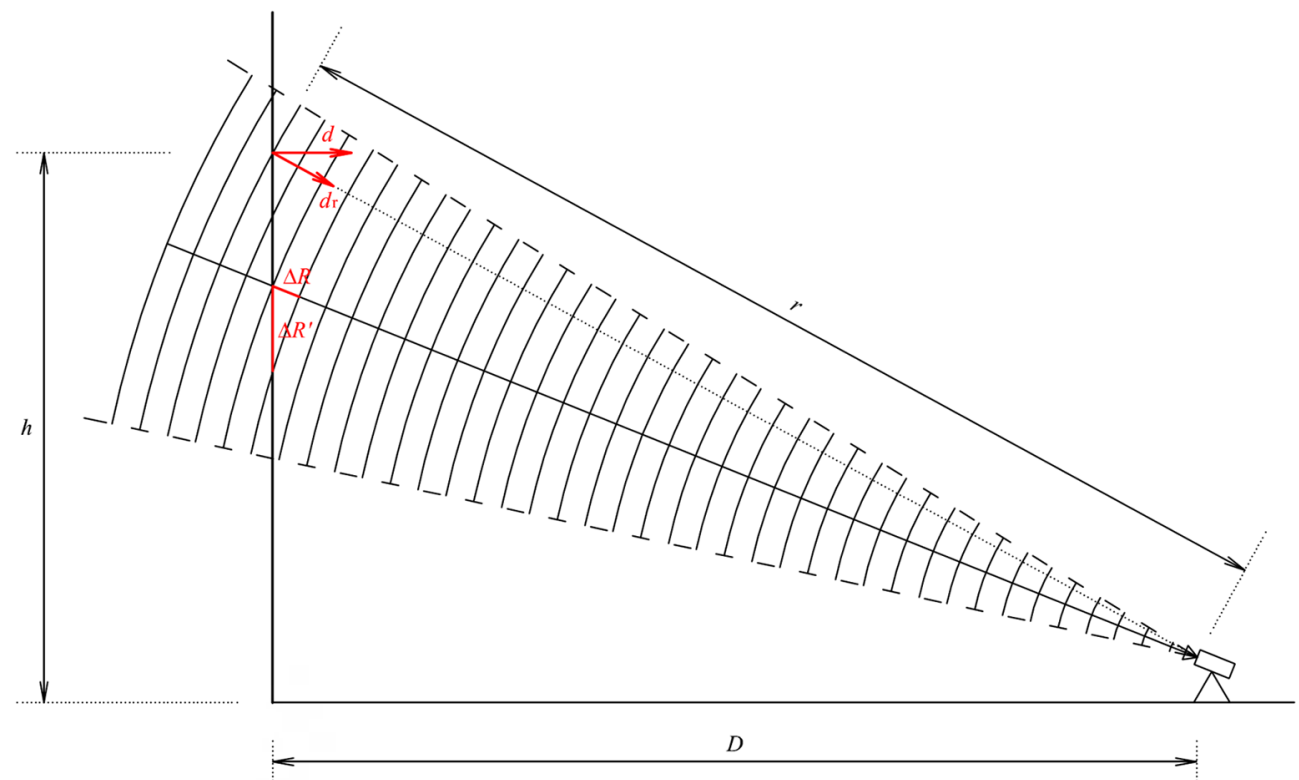

Fig. 2. The relationship between the real and radial displacements, and between the range resolution and the effective resolution during the observations of tall building structures

\subsection{Projection Factor}

The interferometric radar allows for the measurements of the displacements only in one direction - in the direction of wave propagation. If the observed point moves in the other direction (e.g. horizontal by the value of $d$ - as in Figure 2), the value of the radial displacement $d_{r}$ will be measured, i.e. the component of the real displacement vector $d$. In order to make a conversion of the $d_{r}$ value into $d$, it is necessary to assume the direction of the vector $d$. In the case of tall building structures, it is usually the horizontal direction. Furthermore, two out of three values should be known: $h, D, r$, which can be determined by surveying. The conversion shall be performed according to the following formula:

$$
d=d_{r} \frac{r}{D}=d_{r} \frac{r}{\sqrt{r^{2}-h^{2}}}=d_{r} \frac{\sqrt{D^{2}+h^{2}}}{D}
$$


The ratio:

$$
\frac{d}{d_{r}}=\frac{r}{D}=p
$$

is called the projection factor. The higher the observed point is located, the greater the factor $p$ is. On the contrary, as the distance between the radar and the structure increases (that is, with the increase in the distance $D$ ), the factor $p$ decreases, which means that the observed displacement $d_{r}$ approaches the real displacement $d$. The value of the factor $p$ is thus adjusted by setting the radar unit in a specific relationship to the observed structure.

\subsection{Characteristics of the Antennas}

A transmitting antenna is an element of the radar which is responsible for the formation of the transmitted microwave beam. To describe the shape of the beam, the characteristics of the antenna is used, which is presented in the coordinate system (polar or Cartesian) as the distribution of an electric field generated in space. It is usually expressed in two planes - as elevation and azimuth characteristics.

Together with IBIS radar, the manufacturer supplies several types of horn antennas with various properties. The main differentiating factor is the beam width, which can be expressed using the HPBW angle (half power beam width). This is the angle between two directions, for which the radiation power is half as low (i.e. $-3 \mathrm{~dB}$ in a logarithmic scale) as than for the direction of maximum radiation. The characteristics of a sample antenna (IBIS-ANT4), which can be treated as a narrow-angle one, has been presented in Figure 3. HPBW angles (elevation and azimuth) are $10^{\circ}$ and $11^{\circ}$ respectively, while for the wide-angle antennas, they reach the values of $39^{\circ}$ (IBIS-ANT5) and 51 (IBIS-ANT6) [6].

a)

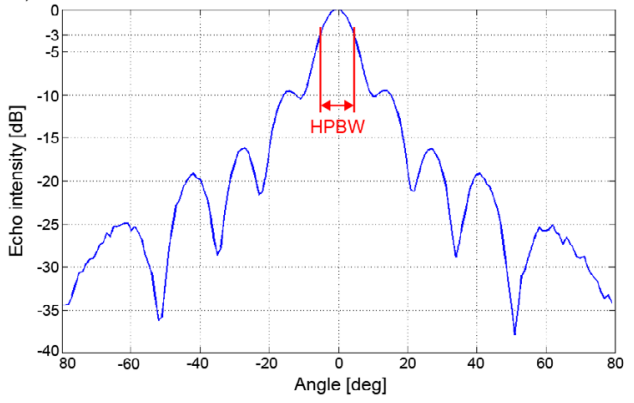

b)

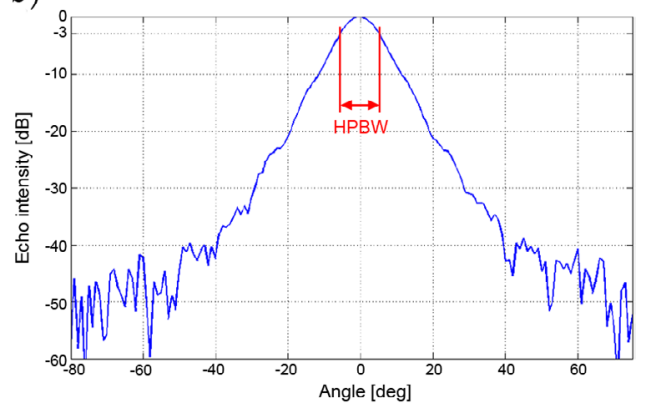

Fig. 3. Characteristics of narrow-angle antenna IBIS-ANT4: a) elevation; b) azimuth Source: [6] 


\section{Planning a Measurement in Terms of Geometry}

In planning observations of high building structures, several factors that affect the efficiency of a measurement should be taken into account. These include:

- effective resolution,

- projection factor,

- range of observation, i.e. which part of the structure is subject to a measurement,

- interdependencies between parameters in the dynamic mode.

\subsection{Effective Resolution}

Effective resolution $\Delta R^{\prime}$ (Fig. 2) determines the number of the observed points on the structure and depends on the position of the instrument relative to the observed structure. In the case of tall structures, the best effective resolution (i.e. the number of points representing the structure) is achieved by setting the radar at the shortest possible distance from the structure $(D \rightarrow 0)$ (Fig. 4).

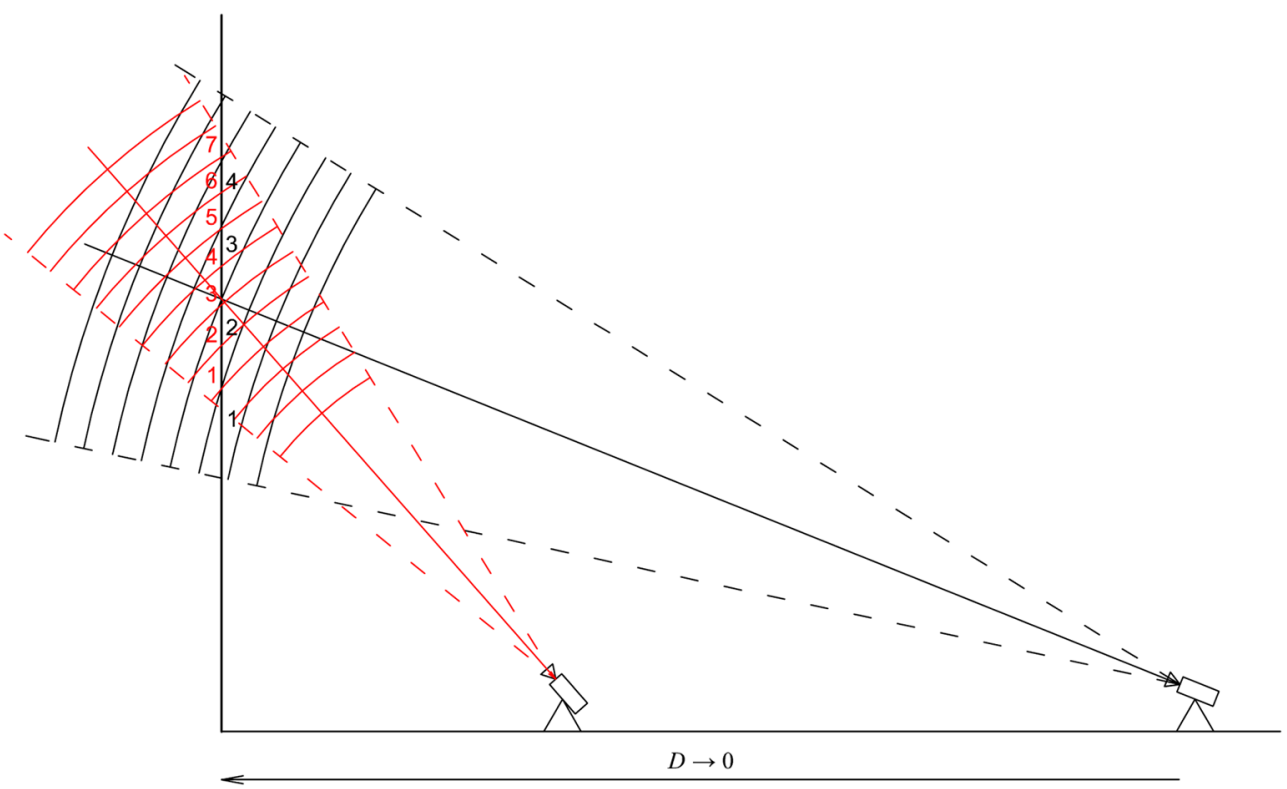

Fig. 4. The effect of setting the radar to the effective resolution

This relationship can be represented by a graph (Fig. 5). For example, the highest fragment of a structure with a height of $h=200 \mathrm{~m}$, observed from a distance of $D=60 \mathrm{~m}$, will be seen with the resolution $\Delta R^{\prime}=0.52 \mathrm{~m}$. However, if the distance $D$ increases to $300 \mathrm{~m}$, then the effective resolution will be worse than $0.90 \mathrm{~m}$. Taking into account the effective resolution, it can therefore be concluded that the most advantageous is to set the instrument in the vicinity of the structure. 


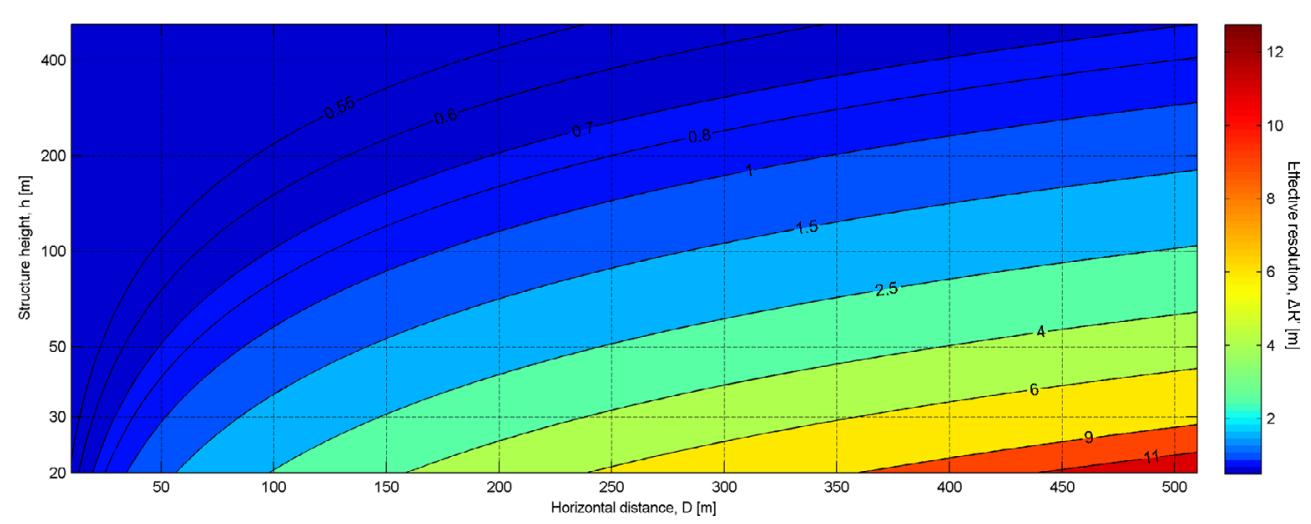

Fig. 5. Dependence of the effective resolution from the radar-structure distance and the height of the structure

\subsection{The Projection Factor}

However, minimizing the distance $D$ is not advantageous as to the accuracy of displacement measurements. The assumed accuracy of displacement measurement using interferometric radar at the level of $0.1 \mathrm{~mm}$ refers to the measured vector $d_{r}$. To estimate the error of horizontal displacement $d$, it is necessary to apply the rule of error transfer in the equation (1), namely:

$$
m_{d}=\sqrt{\left(\frac{\partial d}{\partial d_{r}}\right)^{2} m_{d r}^{2}+\left(\frac{\partial d}{\partial D}\right)^{2} m_{D}^{2}+\left(\frac{\partial d}{\partial h}\right)^{2} m_{h}^{2}}
$$

where:

and:

$m_{d r}$ - displacement measurement error in the radial direction,

$m_{D}$ - error of determining the radar-structure horizontal distance,

$m_{h}$ - error of determining the height of the structure

$$
\left(\frac{\partial d}{\partial d_{r}}\right)^{2}=1+\frac{h^{2}}{D^{2}},\left(\frac{\partial d}{\partial D}\right)^{2}=\frac{\left(D d_{r} h-d_{r}\left(D^{2}+h^{2}\right)\right)^{2}}{D^{4}\left(D^{2}+h^{2}\right)},\left(\frac{\partial d}{\partial h}\right)^{2}=\frac{d_{r}^{2} h^{2}}{D^{2}\left(D^{2}+h^{2}\right)}
$$

The analysis assumes that $m_{d r}=0.1 \mathrm{~mm}$. It was also assumed that the error of determining the horizontal distance and the height of the structure $m_{D}=m_{h}=0.5 \mathrm{~m}$. This value results from the resolution $\Delta R$ of the radar, which means that with such an accuracy a position of the observed point on the structure can be estimated. This factor, however, does not affect significantly the value of $m_{d}$.

The equation (3) can be represented by a graph (Fig. 6). 
Two cases were considered, in which the value of the real horizontal displacement is:

a) $d=1 \mathrm{~mm}$,

b) $d=50 \mathrm{~mm}$.

For example, in the case "a", if the highest point of the structure with a height of $h=200 \mathrm{~m}$ is observed from a distance of $D=300 \mathrm{~m}$, then the horizontal displacement $d=1 \mathrm{~mm}$ will be determined with the error $m_{d}=0.12 \mathrm{~mm}$, taking into account the above assumptions. However, in the case " $b$ ", if this point is observed from a distance of $D=50 \mathrm{~m}$, then the displacement will be $d=50 \mathrm{~mm} \pm 0.57 \mathrm{~mm}$.

a)

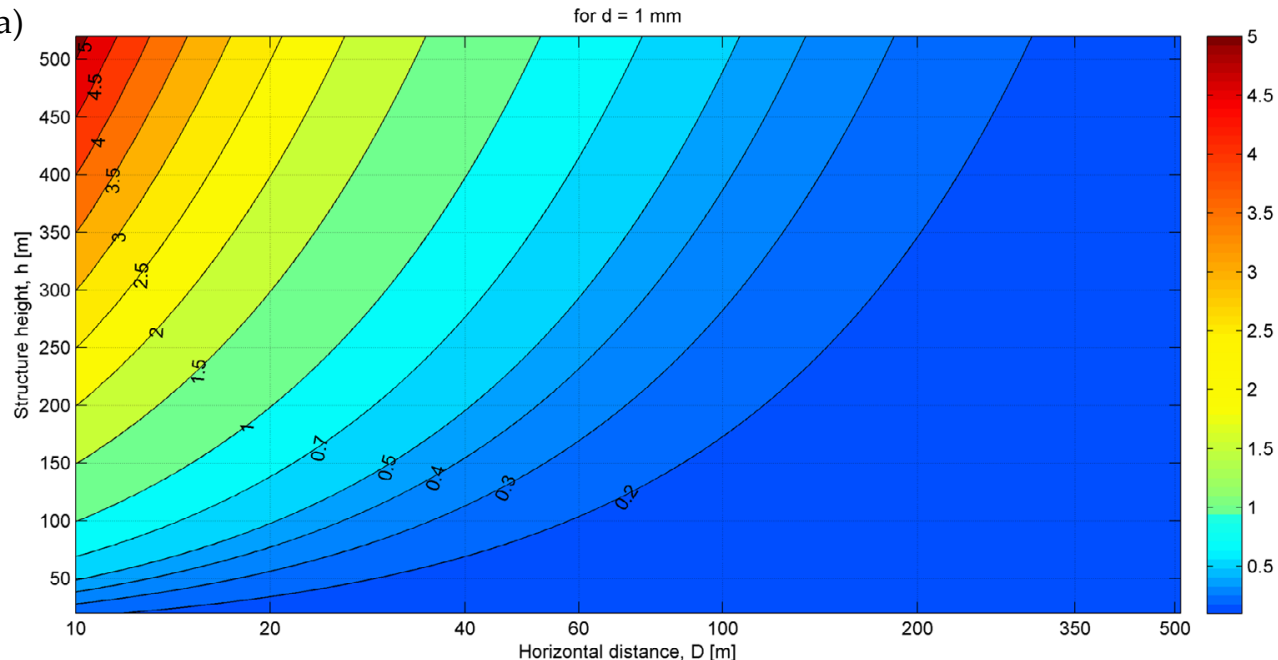

b)

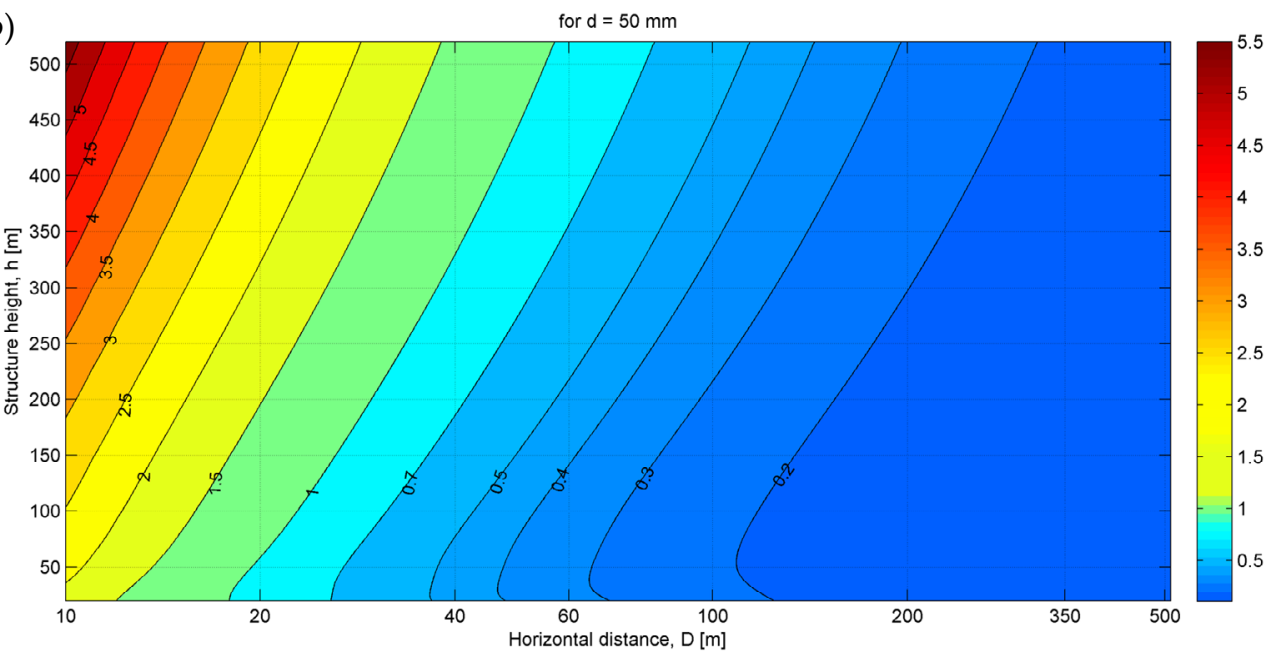

Fig. 6. Dependence of the horizontal displacement error on the radar-structure distance and the height of the structure 


\subsection{The Scope of Observations}

When observing the displacements of tall building structures, the highest point of the structure is usually observed. However, sometimes it is equally important to determine the behavior of the points which are located at lower levels. One of the main advantages of the interferometric radar is the ability to measure a number of points simultaneously. When planning such a measurement, attention should be paid to the range of the space observed by the radar. This range can be expressed using the HPBW angle (Fig. 3). This does not mean that the observation of other points of the structure out of this range is impossible, however the intensity of the wave transmitted in those directions is more than twice lower than in the direction of the radar axis, resulting in a lower SNR of the observed points, and thus in the decrease of the measurement accuracy.

Before setting the instrument, it should be considered, what the length of the observed structure (from the top) shall be subject to the observation. In Figure 7, this section has been marked as $x$. Given the height $h$ of the observed structure, the value of the HPBW angle (designated as $\alpha$ ) and the height of the observation stand $z$ respectively to the base of the structure, the distance $D$ must satisfy the following condition:

$$
D \leq D_{1} \quad \text { or } \quad D \geq D_{2}
$$

where:

$$
D_{1,2}=\frac{x \mp \sqrt{x^{2}-4 \tan ^{2} \alpha(h-z)(h-z-x)}}{2 \tan \alpha}
$$

If determination of real values of $D_{1}$ and $D_{2}$ is impossible, i.e. if:

$$
x<2 \tan \alpha \sqrt{(h-z)(h-z-x)}
$$

then the whole section $x$ is covered by the beam in the HPBW range.

For example, to observe a section of the length of $x=100 \mathrm{~m}$ of a structure with the height of $h=300 \mathrm{~m}$ using antennas IBIS-ANT4 $\left(\alpha=10^{\circ}\right)$, an observation stand should be assumed at a distance $D \leq D_{1}=140.7 \mathrm{~m}$, or $D \geq D_{2}=426.4 \mathrm{~m}$ from the structure (assuming that $z=0$ ). These ranges have been marked in green in Figure 7 . If $z \neq 0$, the values of $D_{1}$ and $D_{2}$ will be subject to a change, according to the example in Figure 7. 


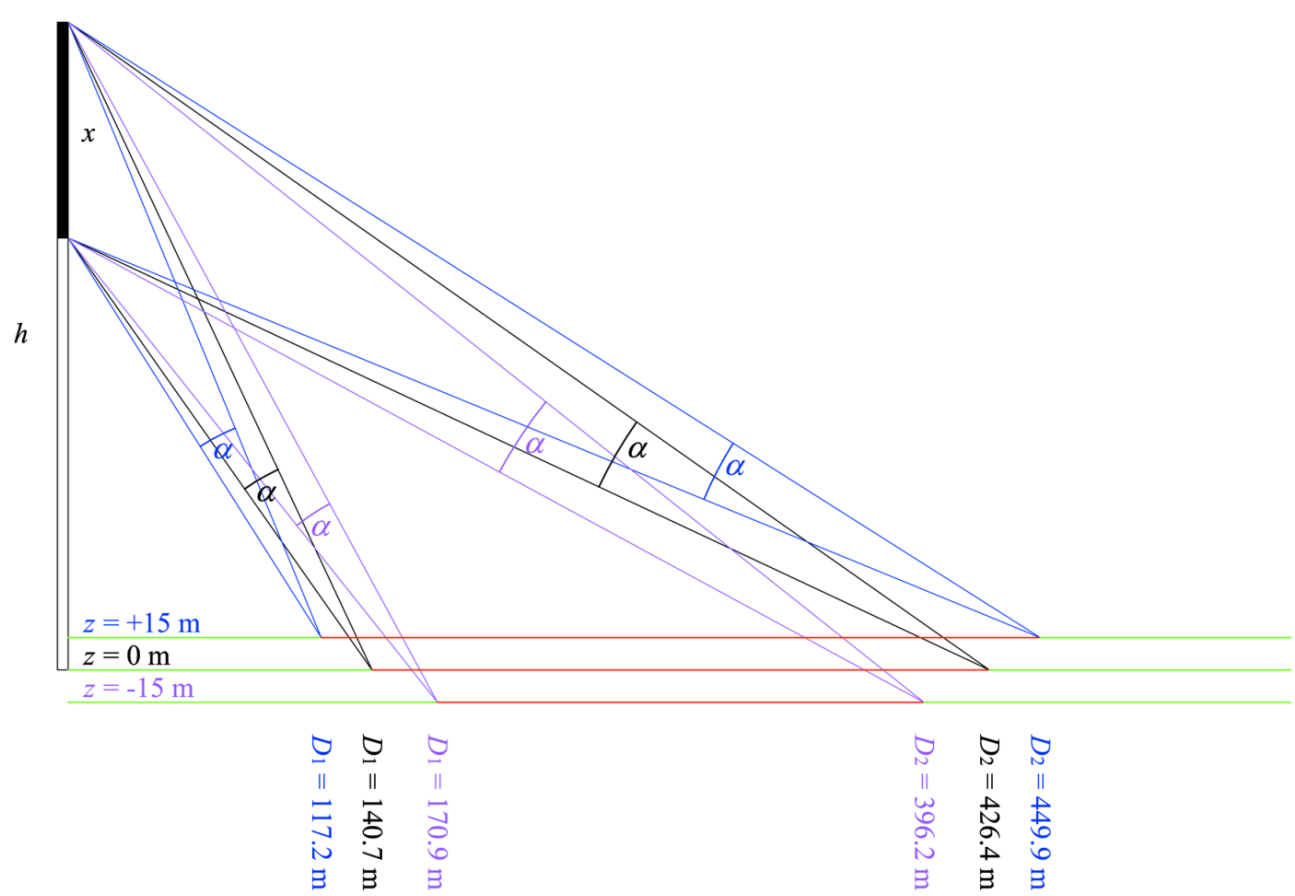

Fig. 7. The method of selecting an observation stand dependent on the range of observation

\subsection{Interdependencies of Parameters in the Dynamic Mode}

The IBIS-S system can operate in either a static or dynamic mode. The latter mode requires to set the following operating parameters:

- the maximum range $R_{\max }$ in the interval $10-2000 \mathrm{~m}$,

- the sampling frequency $f_{s}$ in the interval $1-200 \mathrm{~Hz}$,

- the range resolution $\Delta R$ in the interval $0.5-10 \mathrm{~m}$ (where these are discrete values: $0.5,0.75,1,1.5,2,3,4,5,7.5$ and $10 \mathrm{~m}$ ).

These parameters are interdependent and it is not possible to implement all the maximum values at the same time [3]. Mutual constraints of the parameters have been shown in Figure 8.

For example, if the instrument is set at a distance of $D_{2}=426.4 \mathrm{~m}$ from the structure of the height of $h=300 \mathrm{~m}$, assuming that $z=0$ (Fig. 7), the spatial distance to the top will be $521.4 \mathrm{~m}$. Such a value (or greater) should be set as $R_{\max }$ range. Then, intending to keep $\Delta R=0.5 \mathrm{~m}$, the maximum sampling frequency must be lower than $40 \mathrm{~Hz}$, as shown in Figure 8. On the other hand, intending to achieve in this case the maximum possible frequency $f_{s}=200 \mathrm{~Hz}$, the range resolution must be limited to $\Delta R=3.0 \mathrm{~m}$. 


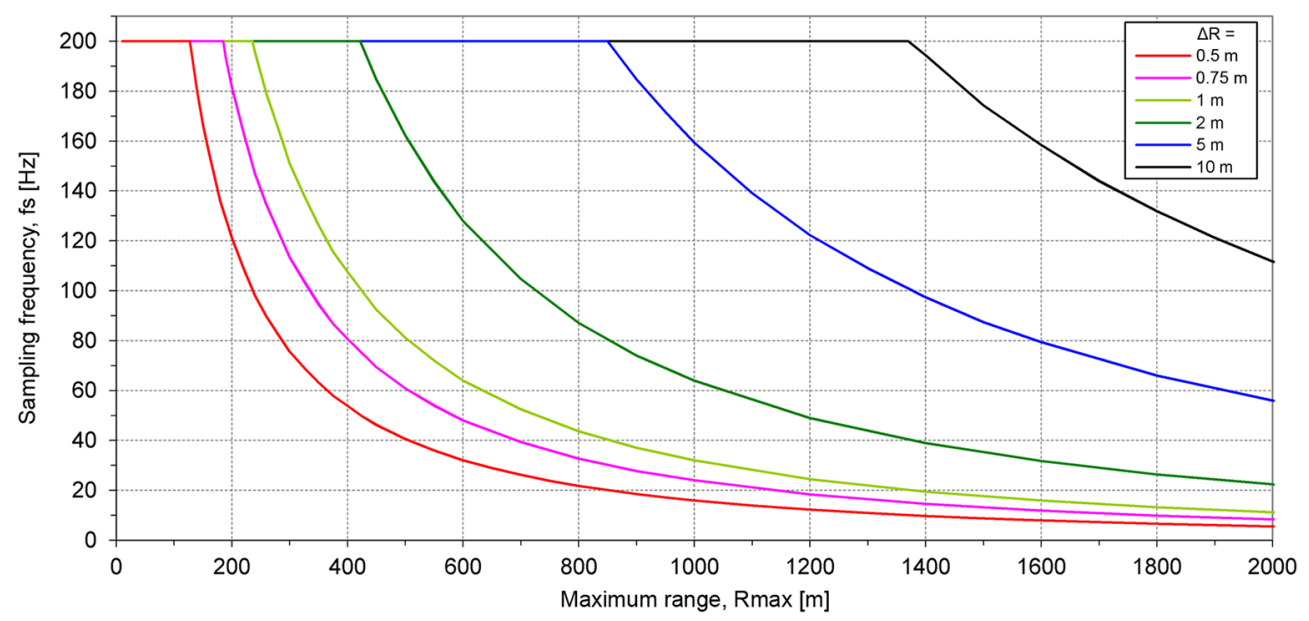

Fig. 8. Interdependencies between the parameters of the radar operation in the dynamic mode

Source: $[3,5]$

\section{The Analysis}

Analysis of the results of the measurements conducted using the IBIS radar system are performed using IBIS Data Viewer software (IBISDV). Its services can be divided into several stages, described in detail in [5]:

- project management,

- data processing,

- selection of the points for analysis,

- analysis of the results.

The program has a variety of tools which are useful for the analysis of measurements of engineering structure displacements. It allows, among others, to enter parameters of radar signal processing, to analyze a radar profile recorded during the measurement and to enter geometric relationships between the position of the radar and the measured structure. It also enables the analysis of selected points in their static and dynamic behavior, including time-frequency analysis (Fig. 9).

As far as measurement geometry is concerned, attention should be paid to the conversion of the radial displacement values $d_{r}$ to horizontal displacements $d$. This operation can be performed using IBSIDV program, on condition of introducing measurement geometry, i.e. the values of $D$ and $h$, based on which the factor $p$ is determined. To analyze the results of a sample measurement of steel observation tower vibrations, IBISDV in 3.4.14 version was used.

Two points were used for the analysis - the strongest reflections visible on the radar profile recorded for the steel observation tower (Fig. 1d). They are located 
at distances of 91.8 and 94.3 meters from the radar. The factors $p$, calculated in the program for these points, are 2.81 and 2.41, respectively (Fig. 9). These values are inconsistent with the equation (2), as with the increasing height of the point on the structure, the factor $p$ should grow up. In addition, the values are incorrect as well. According to the equation (2), they should be 1.07 and 1.10, respectively. This may imply an error in the software used to process the results. Its existence has been presumed in the analysis of the data obtained from the measurement of a tower structure in the dynamic mode, in the Range-bin Dynamic Analysis module (Fig. 9). The correctness of the calculations performed by other software modules was not verified.

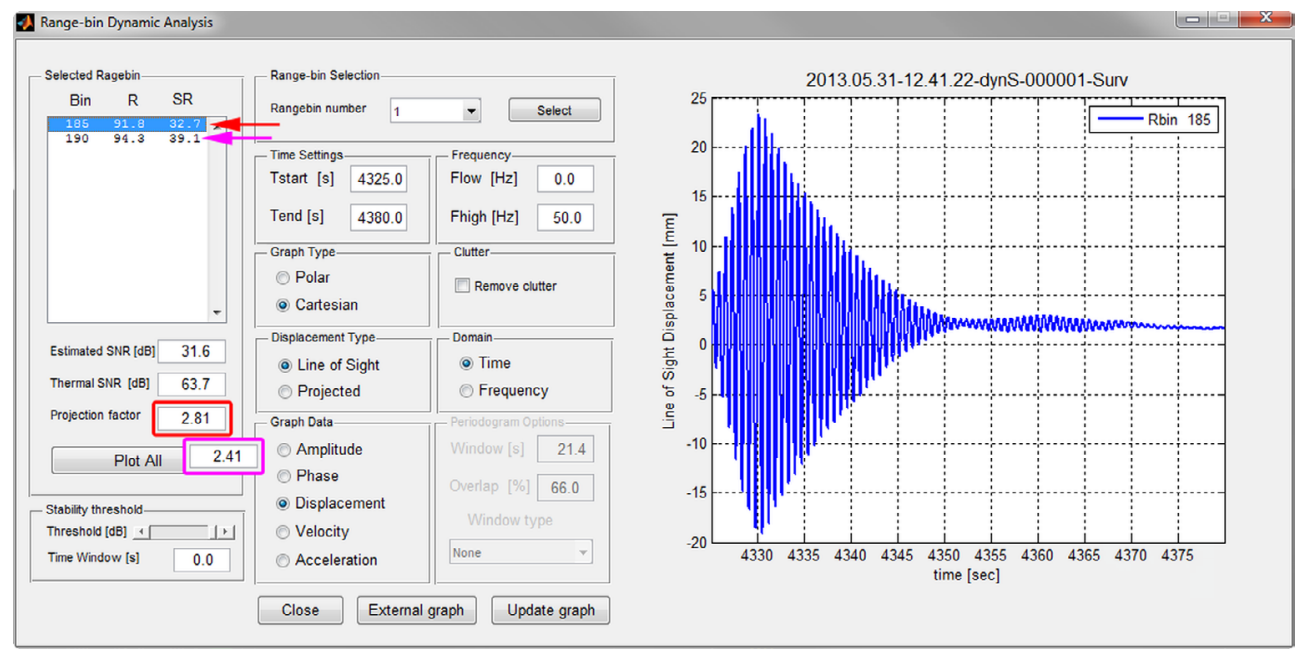

Fig. 9. Calculation of the projection factor in the IBIS Data Viewer software

Incorrect determination of the projection factor affects the calculation of the value of the vector $d$ on the basis of its measured component $d_{r}$. It has been shown as an example based on the data from the measurement presenting the excitation and damping of steel tower vibrations, lasting for 55 seconds. The geometric relationship between the position of the measuring unit IBIS-S and the observed structure has been shown in Figure 1b.

In the analyzed case, the distortion of the real displacements of the test point has been presented in Figure 10. The values of the measured radial displacements $d_{r}$ (along the line of sight) have been marked in black. The maximum amplitude is $21.26 \mathrm{~mm}$. The results of the calculations of the value of $d$ using IBISDV program have been marked in red. The maximum value is $59.70 \mathrm{~mm}$. The values of $d$ calculated in manually are highlighted in green. In this case, the maximum amplitude reaches $22.77 \mathrm{~mm}$. 


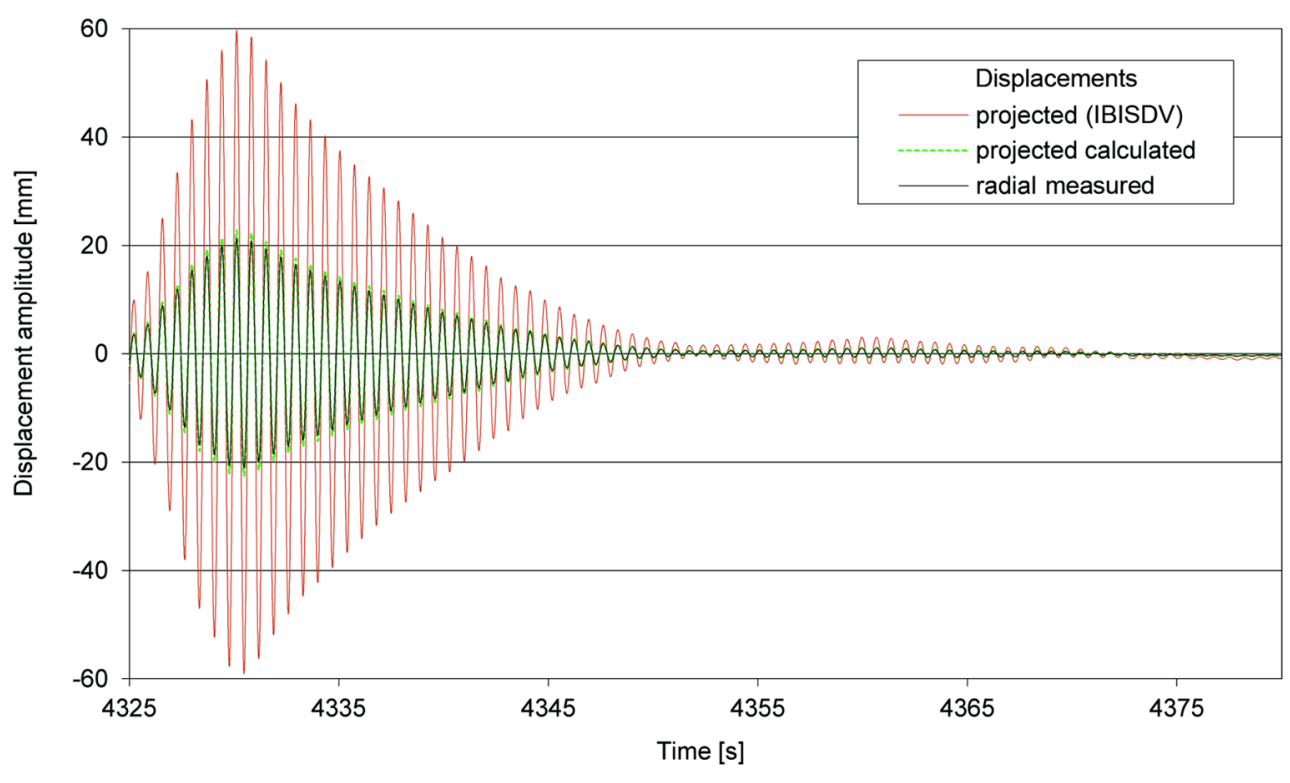

Fig. 10. Incorrect conversion of radial displacements to the real ones

\section{Summary}

Interferometric radar IBIS has many properties which are particularly useful when observing tall building structures. High accuracy of displacement measurements and the possibility to observe the position of multiple points simultaneously, often without a necessity to access the observed structure, have to be mentioned. In addition, the IBIS-S system provides high sampling frequency, allowing for conducting the dynamic tests.

The paper presents geometric aspects related to performing displacement measurements using interferometric radar and the analysis of their results. Particular attention was paid to the issue of planning the measurements. Apparently insignificant setting of the instrument relative to the structure greatly affects the efficiency of the observation.

If it is important to observe a large number of points on the structure, the radar should be placed as close as possible to maximize the effective resolution (Section 4.1). However, it negatively affects the accuracy of a measurement due to a need to convert radial displacement to the real one. The highest accuracy is achieved while conducting observations in the direction of real displacements which, in the case of tall building structures, means moving away from the structure (Section 4.2). A large distance between the instrument and the observed points may, however, cause a decrease in SNR, and thus in the accuracy of the measurement (Chapter 2). 
Other problems occur if the essence of a measurement is the dynamic behavior of the structure. At this point, attention should be paid to the limitation of the radar range and the range resolution by the sampling frequency. In this case, it is advantageous to minimize the radar-structure distance (Section 4.4). Selection of the "intermediate" position, which meets all of the aforementioned criteria, may therefore be associated with some constraints to the scope of observations, resulting from the characteristics of microwave antennas (Section 4.3).

In conclusion, the selection of the radar position is not a simple task. First of all, it must take into account the measurement objective and the resulting constraints.

The article also highlighted the problem of the geometric analysis of observations. Incorrect geometric reduction, which was presumed in one of the IBISDV 3.4.14 software modules will distort the displacement of the points for which the ratio $h / D$ will be different from 1 , causing erroneous inference about the deformation of the studied structure. When analyzing the behavior of a small number of points, this fact may remain unnoticed. The use of other software modules should be preceded by a verification of the correctness of the performed calculations.

\section{References}

[1] Alba M., Bernardini G., Giussani A., Ricci P.P., Roncoroni F., Scaioni M., Valgoi P., Zhang K.: Measurement of dam deformations by terrestrial interferometric techniques. The International Archives of the Photogrammetry, Remote Sensing and Spatial Information Sciences, vol. 37, part B1, 2008, pp. 133-139.

[2] Calcina S.V., Piroddi L., Ranieri G.: Fast dynamic control of damaged historical buildings: a new useful approach for structural health monitoring after an earthquake. ISRN Civil Engineering, vol. 2013, 2013, pp. 1-6.

[3] Gentile C.: Vibration measurement by radar techniques. [in:] EURODYN '11, Leuven, Belgium, July 4-6, 2011, pp. 92-103.

[4] Gikas V.: Ambient vibration monitoring of slender structures by microwave interferometer remote sensing. Journal of Applied Geodesy, vol. 6 (3-4), 2012, pp. 167-176.

[5] Gocał J., Ortyl Ł., Owerko T., Kuras P., Kocierz R., Ćwiąkała P., Puniach E., Sukta O., Bałut A.: Determination of displacements and vibrations of engineering structures using ground-based radar interferometry. Wydawnictwa AGH, Kraków 2013.

[6] IBIS-S System. User Manual. IDS Ingegneria dei Sistemi S.p.A., Pisa 2010.

[7] Kohut P., Holak K., Uhl T., Ortyl Ł., Owerko T., Kuras P., Kocierz R.: Monitoring of a civil structure's state based on noncontact measurements. Structural Health Monitoring, vol. 12(5-6), 2013, pp. 411-429. 
[8] Kopáčik A., Lipták I., Kyrinovič P., Erdélyi J.: Monitoring of a cycling bridge using accelerometers and ground-based radar - a case study. [in:] $2^{\text {nd }}$ Joint International Symposium on Deformation Monitoring, Nottingham, 9-10 September, 2013, pp. 1-8.

[9] Kuras P., Owerko T., Ortyl Ł., Kocierz R., Sukta O., Pradelok S.: Advantages of radar interferometry for assessment of dynamic deformation of bridge. [in:] Bridge Maintenance, Safety, Management, Resilience and Sustainability: Proceedings of the Sixth International IABMAS Conference, Stresa, Lake Maggiore, Italy, 8-12 July 2012, pp. 885-891.

[10] Neitzel F., Niemeier W., Weisbrich S., Lehmann M.: Investigation of low-cost accelerometer, terrestrial laser scanner and ground-based radar interferometer for vibration monitoring of bridges. [in:] $6^{\text {th }}$ European Workshop on Structural Health Monitoring, Dresden, 3-6 July, 2012, pp 1-10.

[11] Owerko T., Ortyl Ł., Kocierz R., Kuras P., Salamak M.: Investigation of displacements of road bridges under test loads using radar interferometry - case study. [in:] Bridge Maintenance, Safety, Management, Resilience and Sustainability: Proceedings of the Sixth International IABMAS Conference, Stresa, Lake Maggiore, Italy, 8-12 July 2012, pp. 181-188.

[12] Owerko T., Ortyl Ł., Kocierz R., Kuras P.: Novel technique of radar interferometry in dynamic control of tall slender structures. Journal of Civil Engineering and Architecture, vol. 6(8), 2012, pp. 1007-1013.

[13] Palombo A., Pignatti S., Perrone A., Soldovieri F., Stabile T.A., Pasucci S.: Noninvasive remote sensing techniques for infrastructures diagnostics. International Journal of Geophysics, vol. 2011, 2011, pp. 1-9.

[14] Pieraccini M., Parrini F., Fratini M., Atzeni C., Spinelli P.: In-Service testing of wind turbine towers using a microwave sensor. Renewable Energy, vol. 33(1), 2008, pp. 13-21.

[15] Pieraccini M., Fratini M., Dei D., Atzeni C.: Structural testing of Historical Heritage Site Towers by microwave remote sensing. Journal of Cultural Heritage, vol. 10(2), 2009, pp. 174-182.

[16] Piniotis G., Mpisis T., Gikas V.: Dynamic testing and output-only modal analysis of a bypass-stack during extreme operating conditions. [in:] $2^{\text {nd }}$ Joint International Symposium on Deformation Monitoring (JISDM) 9-10 September 2013, Nottingham UK, pp. 1-8.

[17] Rödelsperger S., Läufer G., Gerstenecker C., Becker M.: Monitoring of displacements with ground-based microwave interferometry: IBIS-S and IBIS-L. Journal of Applied Geodesy, vol. 4(1), 2010, pp. 41-54.

[18] Rödelsperger S.: Real-time processing of ground based synthetic aperture radar (GBSAR) measurements. Schriftenreihe Fachrichtung Geodäsie, Heft 33, Fachbereich Bauingenieurwesen und Geodäsie, Technische Universität Darmstadt, 2011. 